\title{
Undifferentiated Sex
}

National Cancer Institute

\section{Source}

National Cancer Institute. Undifferentiated Sex. NCI Thesaurus. Code C41438.

Sex could not be determined; not uniquely defined; undifferentiated. 